\title{
On Separation Principle for a Class of Networked Control Systems
}

\author{
Dongxiao $\mathrm{Wu}$, Jun $\mathrm{Wu}$ and Sheng Chen
}

\begin{abstract}
In this contribution, we investigate a class of observer-based discrete-time networked control systems (NCSs) with random packet dropouts occurring independently in both the sensor-to-controller $(\mathrm{S} / \mathrm{C})$ and controller-to-actuator $(\mathrm{C} / \mathrm{A})$ channels. We first propose and prove a separation principle for the general class of NCSs where packet dropouts in the C/A and $\mathrm{S} / \mathrm{C}$ channels are governed by two independent Markov chains, respectively. We then derive necessary and sufficient conditions, in terms of linear matrix inequalities, for synthesis of stabilisation control of a class of NCSs where the C/A channel is driven by a Markov chain while the $S / C$ channel is driven by a Bernoulli process. A numerical example is provided to illustrate the effectiveness of our proposed method.
\end{abstract}

\section{INTRODUCTION}

An important concept in control theory is the separation principle [1]. When the separation principle holds, the controller and the observer in an observer-based control problem can be designed separately. The separation principle has been widely used in optimal control and estimation problems, such as the linear quadratic gaussian (LQG) optimal control [2]. For nonlinear observer-based control problem, the separation principle is also proposed for example by developping a high gain observer approach [3]. Networked control systems (NCSs) have drawn much attention in the control community recently [4]-[7]. An NCS is a control system in which the control loop is closed via a shared communication network. Compared to the point-to-point system connection, the use of an NCS has advantages of low installation cost, reducing system wiring, simple system diagnosis and easy maintenance. However, some inherent shortcomings of NCSs, such as bandwidth constraints, packet dropouts and packet delays, will degrade performance of NCSs or even cause instability.

Packet dropouts, which can randomly occur due to node failures or network congestion, impose one of the most critical problems in NCSs. Stochastic approaches based on the mean square stability [8], [9] are usually adopted to deal with packet dropout. Under such a stochastic approach, the packet-dropout process is typically modelled as a Bernoulli process [5], [6], [10], [11] or a Markov chain [6], [12], [13], and the system can be viewed as a special case of jump linear system. In some other works [14]-[16], NCSs with arbitrary

D.-X. Wu and J. Wu are with State Key Laboratory of Industrial Control Technology, Institute of Cyber-Systems and Control, Zhejiang University, Hangzhou 310027, China. E-mails: \{dxwu,jwu\}@iipc.zju.edu.cn

S. Chen is with School of Electronics and Computer Science, University of Southampton, Highfield, Southampton SO17 1BJ, UK. E-mail: sqc@ecs.soton.ac.uk

This work is supported by the National Natural Science Foundation of China (Grants No.60774001, No.60736021 and No.60721062), the 973 program of China (Grant No.2009CB320603), the 863 program of China (Grant No.2008AA042602), the 111 Project of China (Grant No.B07031), and the UK Royal Society and Royal Academy of Engineering. packet dropouts are modelled as switched systems. Because of its importance to control system analysis and synthesis, separation principle has been studied for some special NCSs with nominal plant. For example, the separation principle is known to hold for the NCSs under TCP-like protocols, where packet dropouts in each communication channel can be modelled as a Bernoulli process [5]. In [7], the authors prove the separation principle for the system with nominal plant, where the network is only located in the sensor-to-controller (S/C) channel. In [17], the author derives the separation principle for the time-varying sampled data system under the assumption that when the plant output is available at the controller, the actuator also receives the related new control data as well. In reality, however, the packets may be lost independently in the S/C and controller-to-actuator (C/A) channels.

To the best of our knowledge, no work to date has proposed the separation principle for NCSs where packet dropouts are driven by two independent Markov chains in the S/C and C/A channels. Hence, the focus of the present work is to investigate the separation principle for such NCSs. Our contributions are two-fold. Firstly, we present and prove a separation principle for observer-based discrete-time NCSs where packet dropouts occur independently in both the S/C and C/A channels. We formulate this generic class of NCSs as the Markovain jump linear system by modelling the behaviours of packet dropouts as independent Markov chains in both the S/C and C/A channels. Secondly, we derive necessary and sufficient conditions for synthesising stabilisation controller for a class of NCSs where packet dropouts in the $\mathrm{C} / \mathrm{A}$ and $\mathrm{S} / \mathrm{C}$ channels are governed by a Markov chain and a Bernoulli process, respectively. These necessary and sufficient conditions are formulated in terms of linear matrix inequalities (LMIs) [18]. An illustrative example is provided to demonstrate our proposed approach.

\section{Notations AND System Description}

The following notational conventions are adopted throughout the discussion. $\mathbb{R}$ stands for the set of real numbers and $\mathbb{N}$ for the set of nonnegative integers, while $\mathbf{W}>0$ indicates that $\mathbf{W}$ is a positive-definite matrix. Furthermore, $\mathbf{I}$ and $\mathbf{0}$ represent the identity and zero matrices, respectively. Given a discrete-time stochastic signal $\mathbf{r}=\{\mathbf{r}(k)\}_{k \in \mathbb{N}}$ with $\mathbf{r}(k)$ a $\mathbb{R}^{r}$-valued random variable, let us define

$$
\|\mathbf{r}\|_{2 s} \triangleq \sqrt{\sum_{k=0}^{\infty} \mathrm{E}\left[\mathbf{r}^{\top}(k) \mathbf{r}(k)\right]},
$$


where $\mathrm{E}[\cdot]$ denotes the expectation. Let $\ell_{2 s}^{r}$ be the set of rs with $\|\mathbf{r}\|_{2 s}<\infty$. For positive integer $M$, further define $\mathcal{M}=\{0,1, \cdots, M-1\}$.

Consider the following stochastic system, denoted as $\hat{F}$

$$
\left\{\begin{array}{l}
\tilde{\mathbf{x}}(k+1)=\tilde{\mathbf{A}}_{\theta(k)} \tilde{\mathbf{x}}(k)+\tilde{\mathbf{B}}_{\theta(k)} \tilde{\mathbf{u}}(k), \quad k \in \mathbb{N} \\
\tilde{\mathbf{y}}(k)=\tilde{\mathbf{C}}_{\theta(k)} \tilde{\mathbf{x}}(k)+\tilde{\mathbf{D}}_{\theta(k)} \tilde{\mathbf{u}}(k),
\end{array}\right.
$$

where $\tilde{\mathbf{x}}(k) \in \mathbb{R}^{\tilde{n}}, \tilde{\mathbf{u}}(k) \in \mathbb{R}^{\tilde{m}}$ and $\tilde{\mathbf{y}}(k) \in \mathbb{R}^{\tilde{q}}$ are the state, input and output vectors, respectively, while $\tilde{\mathbf{A}}_{\theta(k)} \in \mathbb{R}^{\tilde{n} \times \tilde{n}}$, $\tilde{\mathbf{B}}_{\theta(k)} \in \mathbb{R}^{\tilde{n} \times \tilde{m}}, \tilde{\mathbf{C}}_{\theta(k)} \in \mathbb{R}^{\tilde{q} \times \tilde{n}}$ and $\tilde{\mathbf{D}}_{\theta(k)} \in \mathbb{R}^{\tilde{q} \times \tilde{m}}$. The stochastic process $\theta(k)$ is driven by an $M$-valued discretetime Markov chain [19], which takes values from the finite set $\mathcal{M}$. The transition probabilities of $\theta(k)$ are given by $p_{i, j}=\operatorname{Prob}(\theta(k+1)=j \mid \theta(k)=i)$ with $p_{i, j} \geq 0$ and $\sum_{j \in \mathcal{M}} p_{i, j}=1$ for each $i \in \mathcal{M}$. The following result from [8], [13] defines the stochastic stability of $\hat{F}$.

Definition 1: The system $\hat{F}$ defined in (2) is said to be stochastically stable if for $\tilde{\mathbf{u}}(k) \equiv 0, \forall \tilde{\mathbf{x}}(0) \in \mathbb{R}^{\tilde{n}}$ and $\forall \theta(0) \in \mathcal{M}$, we have $\tilde{\mathbf{x}} \in \ell_{2 s}^{\tilde{n}}$.

Lemma 1: The system $\hat{F}$ defined by (2) is stochastically stable if and only if there exist matrices $0<\mathbf{P}_{i} \in \mathbb{R}^{\tilde{n} \times \tilde{n}}$ such that

$$
\mathbf{P}_{i}-\tilde{\mathbf{A}}_{i}^{\top}\left(\sum_{j=0}^{M-1} p_{i, j} \mathbf{P}_{j}\right) \tilde{\mathbf{A}}_{i}>0, \forall i \in \mathcal{M} .
$$

Lemma 2: (The bounded real lemma [13].) If there exist $0<\beta \in \mathbb{R}$ and matrices $0<\mathbf{P}_{i} \in \mathbb{R}^{\tilde{n} \times \tilde{n}}$ such that

$$
\begin{gathered}
{\left[\begin{array}{cc}
\mathbf{P}_{i} & \mathbf{0} \\
\mathbf{0} & \frac{1}{\beta^{2}} \mathbf{I}_{\tilde{m}}
\end{array}\right]-\left[\begin{array}{cc}
\tilde{\mathbf{A}}_{i} & \tilde{\mathbf{B}}_{i} \\
\tilde{\mathbf{C}}_{i} & \tilde{\mathbf{D}}_{i}
\end{array}\right]^{\top}\left[\begin{array}{cc}
\sum_{j=0}^{M-1} p_{i, j} \mathbf{P}_{j} & \mathbf{0} \\
\mathbf{0} & \mathbf{I}
\end{array}\right]} \\
\times\left[\begin{array}{cc}
\tilde{\mathbf{A}}_{i} & \tilde{\mathbf{B}}_{i} \\
\tilde{\mathbf{C}}_{i} & \tilde{\mathbf{D}}_{i}
\end{array}\right]>0, \forall i \in \mathcal{M},
\end{gathered}
$$

then the system $\hat{F}$ of (2) is stochastically stable, and furthermore for $\tilde{\mathbf{x}}(0)=0, \forall \tilde{\mathbf{u}} \in \ell_{2 s}^{\tilde{m}}$ and $\forall \theta(0) \in \mathcal{M}$, we have $\|\tilde{\mathbf{y}}\|_{2 s} \leq\|\tilde{\mathbf{u}}\|_{2 s} / \beta$.

Lemma 3: (Schur complement [18].) Given constant matrices $\mathbf{G}, \mathbf{M}, \mathbf{H}$ of appropriate dimensions where $\mathbf{G}$ and $\mathbf{H}$ are symmetric, then $\mathbf{H}>0$ and $\mathbf{G}+\mathbf{M}^{\top} \mathbf{H}^{-1} \mathbf{M}<0$ if and only if

$$
\left[\begin{array}{cc}
\mathbf{G} & \mathbf{M}^{\top} \\
\mathbf{M} & -\mathbf{H}
\end{array}\right]<0
$$

or equivalently,

$$
\left[\begin{array}{cc}
-\mathbf{H} & \mathbf{M} \\
\mathbf{M}^{\top} & \mathbf{G}
\end{array}\right]<0 \text {. }
$$

The NCS $\hat{P}_{K}$, depicted in Fig. 1, consists of a discretetime plant $\hat{P}$ and a discrete-time controller $\hat{K}$ with the control loop closed via a shared communication network. The plant $\hat{P}$ is described by the state-space equation

$$
\left\{\begin{aligned}
\mathbf{x}(k+1) & =\mathbf{A x}(k)+\mathbf{B} \mathbf{u}(k), \quad k \in \mathbb{N} \\
\mathbf{y}(k) & =\mathbf{C x}(k),
\end{aligned}\right.
$$

where $\mathbf{x}(k) \in \mathbb{R}^{n}, \mathbf{u}(k) \in \mathbb{R}^{m}$ and $\mathbf{y}(k) \in \mathbb{R}^{q}$ are the state, input and output vectors, respectively, while $\mathbf{A} \in \mathbb{R}^{n \times n}$, $\mathbf{B} \in \mathbb{R}^{n \times m}$ and $\mathbf{C} \in \mathbb{R}^{q \times n}$ are constant matrices.
Network packet dropouts occur in both the S/C and C/A channels. Denote the binary set $\mathcal{N} \triangleq\{0,1\}$. Further define $\theta_{s}(k) \in \mathcal{N}$ and $\theta_{a}(k) \in \mathcal{N}$ as the indicators of the packet dropouts in the $\mathrm{S} / \mathrm{C}$ and $\mathrm{C} / \mathrm{A}$ channels, respectively, where a value 0 indicates that the packet is dropped while a value 1 indicates that the packet is transmitted successfully. We make the following generic assumption for these two binary-valued discrete-time random variables.

Assumption 1: $\theta_{a}(k)$ and $\theta_{s}(k)$ are driven by the two independent homogeneous discrete-time Markov chains, which take values in the binary set $\mathcal{N}$ with the transition probability matrices $\boldsymbol{\Lambda} \triangleq\left[\lambda_{i, j}\right]$ and $\boldsymbol{\Pi} \triangleq\left[\pi_{t, r}\right]$, respectively. Here the probabilities $\lambda_{i, j}$ and $\pi_{t, r}$ are defined by

$$
\lambda_{i, j}=\operatorname{Prob}\left(\theta_{a}(k+1)=j \mid \theta_{a}(k)=i\right)
$$

and

$$
\pi_{t, r}=\operatorname{Prob}\left(\theta_{s}(k+1)=r \mid \theta_{s}(k)=t\right),
$$

respectively, with $\lambda_{i, j} \geq 0, \pi_{t, r} \geq 0, \sum_{j \in \mathcal{N}} \lambda_{i, j}=1$ and $\sum_{r \in \mathcal{N}} \pi_{t, r}=1, \forall i, j, t, r \in \mathcal{N}$.

The controller $\hat{K}$, similar to the one in [7], is adopted as

$$
\left\{\begin{aligned}
\hat{\mathbf{x}}(k+1)= & \mathbf{A} \hat{\mathbf{x}}(k)+\mathbf{B u}(k) \\
& +\theta_{s}(k) \mathbf{L}(\mathbf{C} \hat{\mathbf{x}}(k)-\mathbf{y}(k)), \quad k \in \mathbb{N} \\
\mathbf{u}(k)= & \theta_{a}(k) \mathbf{K} \hat{\mathbf{x}}(k),
\end{aligned}\right.
$$

where the state feedback gain matrix $\mathbf{K} \in \mathbb{R}^{m \times n}$ and the observer gain matrix $\mathbf{L} \in \mathbb{R}^{n \times q}$. It can be seen that a standard observer law is employed to estimate $\mathbf{x}(k)$ using $\hat{\mathbf{x}}(k)$ when $\mathbf{y}(k)$ is available at $\theta_{s}(k)=1$, while an imitation law is employed to estimate $\mathbf{x}(k)$ with $\hat{\mathbf{x}}(k)$ when $\mathbf{y}(k)$ is unavailable at $\theta_{s}(k)=0$. Also if the packet is transmitted successfully in the C/A channel at $\theta_{a}(k)=1, \mathbf{u}(k)=$ $\mathbf{K} \hat{\mathbf{x}}(k)$. If however the packet is lost at $\theta_{a}(k)=0$, the actuator does nothing and we have $\mathbf{u}(k)=\mathbf{0}$.

Let us define the state of the NCS $\hat{P}_{K}$ as

$$
\overline{\mathbf{x}}(k) \triangleq\left[\mathbf{x}^{\top}(k) \mathbf{e}^{\top}(k)\right]^{\top},
$$

where $\mathbf{e}(k)=\mathbf{x}(k)-\hat{\mathbf{x}}(k)$. From (3) and (6), the NCS $\hat{P}_{K}$ can be described by

$$
\overline{\mathbf{x}}(k+1)=\overline{\mathbf{A}}_{\theta_{a}(k) \theta_{s}(k)} \overline{\mathbf{x}}(k), \forall k \in \mathbb{N},
$$

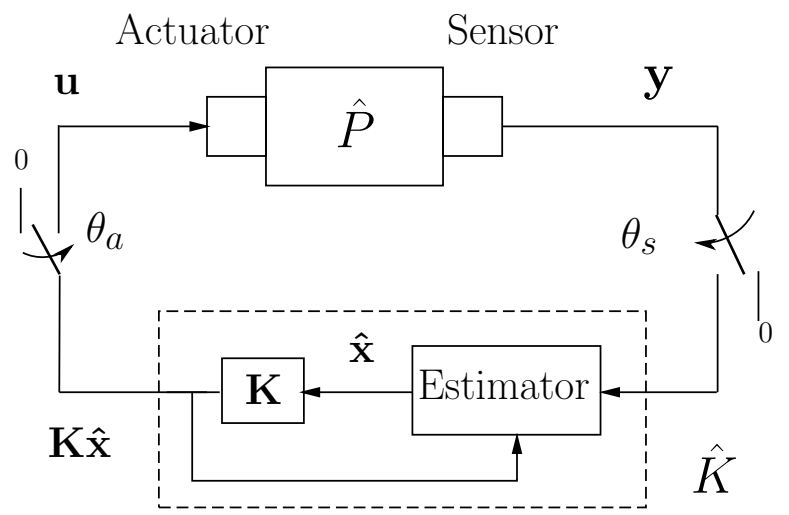

Fig. 1. Networked control system $\hat{P}_{K}$. 
with

$$
\begin{gathered}
\overline{\mathbf{A}}_{00}=\left[\begin{array}{cc}
\mathbf{A} & \mathbf{0} \\
\mathbf{0} & \mathbf{A}
\end{array}\right], \overline{\mathbf{A}}_{01}=\left[\begin{array}{cc}
\mathbf{A} & \mathbf{0} \\
\mathbf{0} & \mathbf{A}+\mathbf{L C}
\end{array}\right], \\
\left\{\begin{array}{l}
\overline{\mathbf{A}}_{10}=\left[\begin{array}{cc}
\mathbf{A}+\mathbf{B K} & -\mathbf{B K} \\
\mathbf{0} & \mathbf{A}
\end{array}\right], \\
\overline{\mathbf{A}}_{11}=\left[\begin{array}{cc}
\mathbf{A}+\mathbf{B K} & -\mathbf{B K} \\
\mathbf{0} & \mathbf{A}+\mathbf{L C}
\end{array}\right] .
\end{array}\right.
\end{gathered}
$$

We can now make the following two observations regarding our NCS model.

Remark 1: We assume that packet dropouts in the C/A and S/C channels are governed by two independent random processes. This assumption is more realistic than the one given in [17] where the connections for the C/A and S/C channels are assumed to be on or off at the same time. In addition, the discrete-time system is more suitable for modelling the NCS than the assumption of sampled-data system in [17], due to the fact that data are transmitted in the form of packets.

Remark 2: We study the generic case where packet dropouts occur in both the C/A and S/C channels independently, while in the work of [7] the network is only located in the S/C channel. Furthermore, necessary and sufficient conditions for separation principle are derived in this paper, while the work [7] only provided sufficient results.

\section{SeParation PRinciple}

In this section, we develop the separation principle for the generic NCS with packet dropouts, which can then be applied to analyse and design the state feedback gain matrix and the observer gain matrix, separately. It is noted that the NCS $\hat{P}_{K}$ defined in (8) is formulated as a Markovian jump linear system, and we prove our separation principle in the following theorem.

Theorem 1: The NCS $\hat{P}_{K}$ (8) under Assumption 1 is stochastically stable if and only if the following two conditions hold.

(i) there exist matrices $\mathbf{Q}_{0}>0$ and $\mathbf{Q}_{1}>0$ such that

$$
\left\{\begin{array}{l}
\hat{\mathbf{A}}_{0}^{\top}\left(\pi_{0,0} \mathbf{Q}_{0}+\pi_{0,1} \mathbf{Q}_{1}\right) \hat{\mathbf{A}}_{0}-\mathbf{Q}_{0}<0 \\
\hat{\mathbf{A}}_{1}^{\top}\left(\pi_{1,0} \mathbf{Q}_{0}+\pi_{1,1} \mathbf{Q}_{1}\right) \hat{\mathbf{A}}_{1}-\mathbf{Q}_{1}<0
\end{array}\right.
$$

where

$$
\hat{\mathbf{A}}_{0}=\mathbf{A}, \hat{\mathbf{A}}_{1}=\mathbf{A}+\mathbf{L C} ;
$$

(ii) there exist matrices $\mathbf{P}_{0}>0$ and $\mathbf{P}_{1}>0$ such that

$$
\left\{\begin{array}{l}
\boldsymbol{\Phi}_{0}^{\top}\left(\lambda_{0,0} \mathbf{P}_{0}+\lambda_{0,1} \mathbf{P}_{1}\right) \boldsymbol{\Phi}_{0}-\mathbf{P}_{0}<0 \\
\boldsymbol{\Phi}_{1}^{\top}\left(\lambda_{1,0} \mathbf{P}_{0}+\lambda_{1,1} \mathbf{P}_{1}\right) \boldsymbol{\Phi}_{1}-\mathbf{P}_{1}<0
\end{array}\right.
$$

where

$$
\mathbf{\Phi}_{0}=\mathbf{A}, \boldsymbol{\Phi}_{1}=\mathbf{A}+\mathbf{B K} .
$$

Proof: (If). From (7) to (12), a subsystem for e can readily be obtained as

$$
\mathbf{e}(k+1)=\hat{\mathbf{A}}_{\theta_{s}(k)} \mathbf{e}(k), \forall k \in \mathbb{N} .
$$

According to Lemma 1 and (11), the subsystem (15) is stochastically stable. Hence, we obtain e $\in \ell_{2 s}^{n}$. From (7) to (14), a subsystem for $\mathrm{x}$ can readily be obtained as

$$
\mathbf{x}(k+1)=\boldsymbol{\Phi}_{\theta_{a}(k)} \mathbf{x}(k)+\boldsymbol{\Gamma}_{\theta_{a}(k)} \mathbf{e}(k),
$$

where

$$
\Gamma_{0}=\mathbf{0}, \Gamma_{1}=-\mathrm{BK}
$$

Partition $\mathbf{x}$ as

$$
\mathbf{x}(k)=\mathbf{x}_{a}(k)+\mathbf{x}_{b}(k),
$$

with

$$
\begin{gathered}
\mathbf{x}_{a}(k+1)=\boldsymbol{\Phi}_{\theta_{a}(k)} \mathbf{x}_{a}(k), \mathbf{x}_{a}(0)=\mathbf{x}(0) ; \\
\mathbf{x}_{b}(k+1)=\boldsymbol{\Phi}_{\theta_{a}(k)} \mathbf{x}_{b}(k)+\boldsymbol{\Gamma}_{\theta_{a}(k)} \mathbf{e}(k), \mathbf{x}_{b}(0)=0 .
\end{gathered}
$$

From Lemma 1 and Definition 1 , it is easy to show $\mathbf{x}_{a} \in$ $\ell_{2 s}^{n}$. If (13) is satisfied, then there exists a sufficiently small constant $0<\rho \in \mathbb{R}$ such that

$$
\begin{aligned}
\mathbf{P}_{i}-\boldsymbol{\Phi}_{i}^{\top} \hat{\mathbf{P}}_{i} \boldsymbol{\Phi}_{i}-\rho^{2} \mathbf{I}-\boldsymbol{\Phi}_{i}^{\top} \hat{\mathbf{P}}_{i} \boldsymbol{\Gamma}_{i}\left(\frac{1}{\rho^{2}} \mathbf{I}-\boldsymbol{\Gamma}_{i}^{\top} \hat{\mathbf{P}}_{i} \boldsymbol{\Gamma}_{i}\right)^{-1} \\
\times \boldsymbol{\Gamma}_{i}^{\top} \hat{\mathbf{P}}_{i} \boldsymbol{\Phi}_{i}>0
\end{aligned}
$$

for $i \in \mathcal{N}$, where $\hat{\mathbf{P}}_{i} \triangleq \sum_{j \in \mathcal{N}} \lambda_{i, j} \mathbf{P}_{j}$. According to Lemma 3, the algebraic inequality (19) is equivalent to the following matrix inequality

$$
\begin{gathered}
{\left[\begin{array}{cc}
\mathbf{P}_{i} & \mathbf{0} \\
\mathbf{0} & \frac{1}{\rho^{2}} \mathbf{I}
\end{array}\right]-\left[\begin{array}{cc}
\boldsymbol{\Phi}_{i} & \boldsymbol{\Gamma}_{i} \\
\rho \mathbf{I} & \mathbf{0}
\end{array}\right]^{\top}\left[\begin{array}{cc}
\hat{\mathbf{P}}_{i} & \mathbf{0} \\
\mathbf{0} & \mathbf{I}
\end{array}\right]} \\
\times\left[\begin{array}{cc}
\boldsymbol{\Phi}_{i} & \boldsymbol{\Gamma}_{i} \\
\rho \mathbf{I} & \mathbf{0}
\end{array}\right]>0 .
\end{gathered}
$$

This together with Lemma 2 yields

$$
\left\|\rho \mathbf{x}_{b}\right\|_{2 s}<\frac{1}{\rho}\|\mathbf{e}\|_{2 s},
$$

namely, $\mathbf{x}_{b} \in \ell_{2 s}^{n}$. Thus, $\mathbf{x} \in \ell_{2 s}^{n}$. According to Definition 1, the NCS $\hat{P}_{K}$ is stochastically stable.

(Only If). Because the NCS $\hat{P}_{K}$ is stochastically stable, from Definition 1 and the expression of $\overline{\mathbf{x}}(k)$ in (7), it is easy to show $\mathbf{x} \in \ell_{2 s}^{n}$ and $\mathbf{e} \in \ell_{2 s}^{n}$. Thus, the subsystem (15) for e is stochastically stable, and the condition (i) holds according to Lemma 1 . Because $\hat{P}_{K}$ is stochastically stable, it is also clear that for $\mathbf{e}(0)=0$ and $\forall \mathbf{x}(0) \in \mathbb{R}^{n}$, there exists $\mathbf{x} \in \ell_{2 s}^{n}$. On the other hand, for the subsystem (15), $\mathbf{e}(k) \equiv 0$ when $\mathbf{e}(0)=0$. In the case of $\mathbf{e}(0)=0$, the subsystem (16) for $\mathbf{x}$ becomes $\mathbf{x}(k+1)=\boldsymbol{\Phi}_{\theta_{a}(k)} \mathbf{x}(k)$ and is stochastically stable. It is then obvious that the condition (ii) holds. This completes the proof.

It is noted that (11) only involves $\mathbf{L}$ while (13) only involves $\mathbf{K}$. Hence the state feedback control and observer can be designed independently by assuring each separated part be stochastically stable. This property is in accordance with the separation principle. 


\section{Stabilisation Control For a Class of NCSS}

In this section, we study the separation principle for a class of NCSs where packet dropouts are driven by a Markov chain in the C/A channel and a Bernoulli process in the S/C channel, respectively. The synthesis of stabilisation control is derived for this class of NCSs in the form of LMIs. First, our assumption regarding this class of NCSs is formally made.

Assumption 2: $\theta_{a}(k)$ is driven by a discrete-time Markov chain, which takes values from the binary set $\mathcal{N}$ with the transition probabilities $\lambda_{i, j}$ defined in (4). $\theta_{s}(k)$ is driven by a Bernoulli process, which takes values from the binary set $\mathcal{N}$ with the two state probabilities, $\operatorname{Prob}\left(\theta_{s}(k)=1\right)=\alpha$ and $\operatorname{Prob}\left(\theta_{s}(k)=0\right)=1-\alpha$.

The Bernoulli process $\theta_{s}(k)$ can be viewed as a special case of Markov chain, with $\pi_{0,1}=\pi_{1,1}=\alpha$ and $\pi_{1,0}=$ $\pi_{0,0}=1-\alpha$ in (5). In this case, Lemma 1, which presents the stability conditions of Markovian jump linear systems, is equivalent to the following lemma (See [9]).

Lemma 4: If $\theta_{s}(k)$ is driven by a Bernoulli process with $\operatorname{Prob}\left(\theta_{s}(k)=1\right)=\alpha$ and $\operatorname{Prob}\left(\theta_{s}(k)=0\right)=1-\alpha$, then the subsystem (15) is stochastically stable if and only if there exists a matrix $\mathbf{Q}>0$ such that

$$
\alpha(\mathbf{A}+\mathbf{L C})^{\top} \mathbf{Q}(\mathbf{A}+\mathbf{L C})+(1-\alpha) \mathbf{A}^{\top} \mathbf{Q A}-\mathbf{Q}<0 . \text { (22) }
$$

According to Theorem 1 and Lemma 4, we have the following separation principle for the underlying NCS.

Theorem 2: The NCS $\hat{P}_{K}$ (8) under Assumption 2 is stochastically stable if and only if the following two conditions hold.

(i) there exists a matrix $\mathbf{Q}>0$ such that (22) holds;

(ii) there exist matrices $\mathbf{P}_{0}>0$ and $\mathbf{P}_{1}>0$ such that (13) holds.

The synthesis of stabilisation control in the form of LMIs for this class of NCSs is presented in the following theorem.

Theorem 3: The NCS $\hat{P}_{K}$ (8) under Assumption 2 is stochastically stable if and only if there exist matrices $0<$ $\overline{\mathbf{P}}_{0} \in \mathbb{R}^{n \times n}, 0<\overline{\mathbf{P}}_{1} \in \mathbb{R}^{n \times n}, \mathbf{X} \in \mathbb{R}^{m \times n}, 0<\mathbf{Q} \in \mathbb{R}^{n \times n}$ and $\mathbf{Y} \in \mathbb{R}^{n \times q}$ such that the following LMIs are satisfied

$$
\begin{gathered}
{\left[\begin{array}{ccc}
-\overline{\mathbf{P}}_{0} & * & * \\
\sqrt{\lambda_{0,0}} \mathbf{A} \overline{\mathbf{P}}_{0} & -\overline{\mathbf{P}}_{0} & * \\
\sqrt{\lambda_{0,1}} \mathbf{A} \overline{\mathbf{P}}_{0} & \mathbf{0} & -\overline{\mathbf{P}}_{1}
\end{array}\right]<0,} \\
{\left[\begin{array}{ccc}
-\overline{\mathbf{P}}_{1} & * & * \\
\sqrt{\lambda_{1,0}}\left(\mathbf{A} \overline{\mathbf{P}}_{1}+\mathbf{B X}\right) & -\overline{\mathbf{P}}_{0} & * \\
\sqrt{\lambda_{1,1}}\left(\mathbf{A} \overline{\mathbf{P}}_{1}+\mathbf{B X}\right) & \mathbf{0} & -\overline{\mathbf{P}}_{1}
\end{array}\right]<0,} \\
{\left[\begin{array}{ccc}
-\mathbf{Q} & * & * \\
\sqrt{\alpha}(\mathbf{Q} \mathbf{A}+\mathbf{Y C}) & -\mathbf{Q} & * \\
\sqrt{1-\alpha} \mathbf{Q A} & \mathbf{0} & -\mathbf{Q}
\end{array}\right]<0,}
\end{gathered}
$$

where the notation $*$ within a matrix denotes symmetric entries. In this case, the state feedback and observer gains are given by $\mathbf{K}=\mathbf{X} \overline{\mathbf{P}}_{1}^{-1}$ and $\mathbf{L}=\mathbf{Q}^{-1} \mathbf{Y}$, respectively.

Proof: Set $\mathbf{P}_{0}=\overline{\mathbf{P}}_{0}^{-1}, \mathbf{P}_{1}=\overline{\mathbf{P}}_{1}^{-1}$ and $\mathbf{X}=\mathbf{K} \overline{\mathbf{P}}_{1}$. By pre- and post- multiplying (23) and (24) with $\operatorname{diag}\left\{\mathbf{P}_{0}, \mathbf{I}, \mathbf{I}\right\}$ and $\operatorname{diag}\left\{\mathbf{P}_{1}, \mathbf{I}, \mathbf{I}\right\}$, respectively, and applying Lemma 3, we obtain that (23) and (24) are equivalent to (13). Similarly, set $\mathbf{Y}=\mathrm{QL}$ in (25). Then by pre- and post- multiplying
(25) with $\operatorname{diag}\left\{\mathbf{I}, \mathbf{Q}^{-1}, \mathbf{Q}^{-1}\right\}$ and applying Lemma 3, we can see that (25) is equivalent to (22). This completes the proof according to Theorem 2.

\section{Numerical ExAMPLE}

To illustrate the effectiveness of the proposed stabilisation approach, we considered an unstable 4th-order NCS $\hat{P}_{K}(8)$ with the plant parameters

$$
\begin{gathered}
\mathbf{A}=\left[\begin{array}{cccc}
1.4 & 1 & 1 & -1.1 \\
-1.3 & -0.9 & 0.5 & 0.5 \\
0.3 & -0.2 & -1 & 0 \\
-0.5 & -0.3 & -0.5 & -1
\end{array}\right] \\
\mathbf{B}=\left[\begin{array}{cc}
-0.7 & -1 \\
0 & -0.9 \\
0.8 & 0.6 \\
0.1 & 0
\end{array}\right] \\
\mathbf{C}=\left[\begin{array}{llll}
-1 & 0.6 & -0.3 & 0 \\
0.5 & 0.5 & 0.2 & -1
\end{array}\right]
\end{gathered}
$$

The eigenvalues of the plant were $1.1783,-1.1818$ and $-0.7482 \pm 0.5171 i$. Assume that $\theta_{a}(k)$ was driven by the Markov chain with the transition probability matrix

$$
\boldsymbol{\Lambda}=\left[\begin{array}{ll}
0.6 & 0.4 \\
0.2 & 0.8
\end{array}\right],
$$

while $\theta_{s}(k)$ was driven by a Bernoulli process with $\alpha=0.7$.

By applying the Matlab LMI Control Toolbox to solve the LMIs (23) to (25) we obtained the following solution

$$
\begin{gathered}
\overline{\mathbf{P}}_{0}=\left[\begin{array}{cccc}
5.3812 & -6.5861 & -0.2150 & 0.6313 \\
-6.5861 & 17.6187 & -5.0160 & -0.9736 \\
-0.2150 & -5.0160 & 4.1209 & -0.3005 \\
0.6313 & -0.9736 & -0.3005 & 0.6456
\end{array}\right], \\
\overline{\mathbf{P}}_{1}=\left[\begin{array}{cccc}
10.7226 & -12.6823 & 0.2978 & -0.5937 \\
-12.6823 & 26.2139 & -6.0030 & 1.8370 \\
0.2978 & -6.0030 & 16.7452 & -6.2727 \\
-0.5937 & 1.8370 & -6.2727 & 4.1789
\end{array}\right],
\end{gathered}
$$

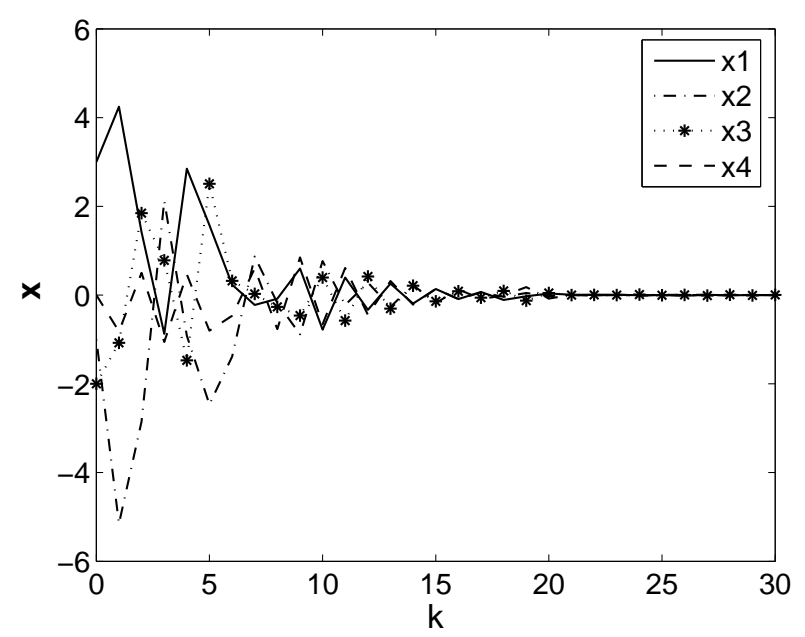

Fig. 2. State trajectories of the plant $\hat{P}$. 


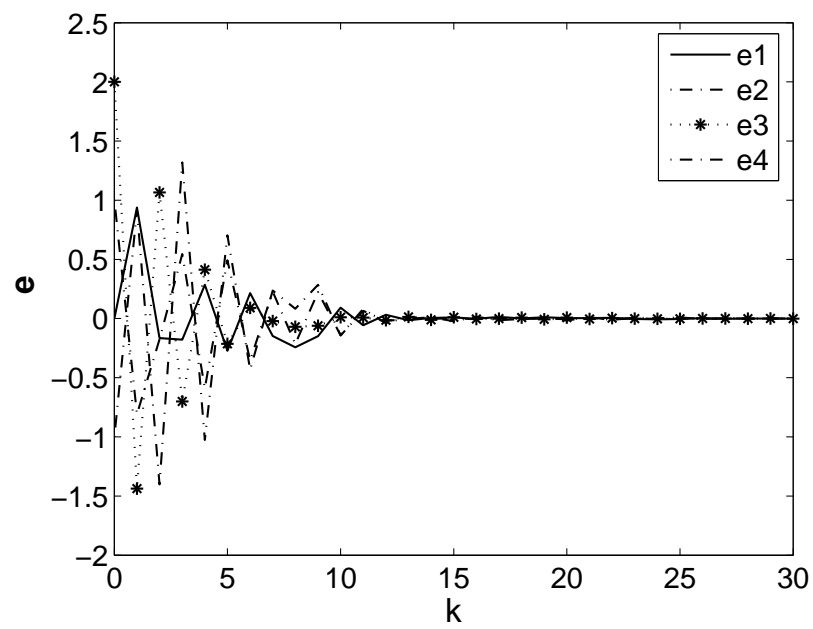

Fig. 3. Error trajectories between the state and the estimator of the NCS $\hat{P}_{K}$.

$$
\begin{gathered}
\mathbf{X}=\left[\begin{array}{cccc}
-4.9778 & 9.2331 & 9.9654 & -1.0929 \\
3.9401 & -7.5202 & 11.0640 & -6.5239
\end{array}\right], \\
\mathbf{Q}=\left[\begin{array}{cccc}
1.4223 & 0.8024 & 0.5724 & 0.2323 \\
0.8024 & 1.2810 & 1.2210 & -0.1602 \\
0.8024 & 1.2810 & 1.2210 & -0.1602 \\
0.2323 & -0.1602 & -0.4595 & 1.5936
\end{array}\right], \\
\mathbf{Y}=\left[\begin{array}{cc}
0.5081 & -1.1919 \\
0.4242 & 0.2342 \\
0.3786 & 0.2348 \\
-0.1656 & -1.3929
\end{array}\right]
\end{gathered}
$$

It followed from Theorem 3 that the synthesis of stochastic stabilisation control was solvable with the state feedback gain matrix and observer gain matrix given respectively by

$$
\begin{gathered}
\mathbf{K}=\mathbf{X} \overline{\mathbf{P}}_{1}^{-1}=\left[\begin{array}{cccc}
0.6786 & 0.9181 & 1.5972 & 1.8288 \\
0.2455 & -0.0407 & 0.1753 & -1.2452
\end{array}\right] \\
\mathbf{L}=\mathbf{Q}^{-1} \mathbf{Y}=\left[\begin{array}{cc}
0.3124 & -1.2852 \\
0.1239 & 1.2140 \\
-0.0061 & -0.3237 \\
-0.1387 & -0.6579
\end{array}\right] .
\end{gathered}
$$

Next, we used the above designed $\mathbf{K}$ and $\mathbf{L}$ to analyse the stability of the NCS $\hat{P}_{K}$. The initial state was chosen to be

$$
\begin{gathered}
\mathbf{x}(0)=\left[\begin{array}{llll}
x_{1}(0) & x_{2}(0) & x_{3}(0) & x_{4}(0)
\end{array}\right]^{\top}=\left[\begin{array}{llll}
3 & -1 & -2 & 0
\end{array}\right]^{\top}, \\
\mathbf{e}(0)=\left[\begin{array}{llll}
e_{1}(0) & e_{2}(0) & e_{3}(0) & e_{4}(0)
\end{array}\right]^{\top}=\left[\begin{array}{llll}
0 & -1 & 2 & 1
\end{array}\right]^{\top},
\end{gathered}
$$

and the initial modes of the two driving stochastic processes were $\theta_{a}(0)=1$ and $\theta_{s}(0)=1$, respectively. Typical response of the NCS $\hat{P}_{K}$ are given in Figs. 2 and 3, which depict the state trajectories of the plant $\hat{P}, \mathbf{x}(k)$, and the error trajectories between the state and the estimator, $\mathbf{e}(k)$, respectively. It can be seen that the NCS $\hat{P}_{K}$ was stochastically stable.

\section{CONCLUSiOnS}

In this contribution we have studied a class of NCSs where random packet dropouts occur independently in both the S/C and C/A channels. Our new contributions have been twofold. Firstly, we have established and proved a separation principle for the generic NCS where packet dropouts are driven by two independent Markov chains for the $\mathrm{S} / \mathrm{C}$ and C/A channels, respectively. According to this separation principle, the state feedback controller and observer for the underlying NCS can be analysed and synthesised separately. Secondly, we have presented the LMI solution that stabilises a class of NCSs where packet dropouts in the C/A and S/C channels are governed by a Markov chain and a Bernoulli process, respectively. A numerical example has been included to illustrate our proposed design approach.

\section{REFERENCES}

[1] P. J. Antsaklis and A. N. Michel, Linear Systems. Boston, MA: Spinger, 2006.

[2] P. S. Maybeck, Stochastic Models, Estimation, and Control. New York: Academic Press, 1979.

[3] A. N. Atassi and H. K. Khalil, "A separation principle for the stabilization of a class of nonlinear systems," IEEE Trans. Automatic Control, vol. 44, pp. 1672-1687, September 1999.

[4] J. P. Hespanha, P. Naghshtabrizi and Y.-G. Xu, "A survey of recent results in networked control systems," Proc. IEEE, vol. 95, pp. 138162, January 2007.

[5] L. Schenato, B. Sinopoli, M. Franceschetti, K. Poolla and S. S. Sastry, "Foundations of control and estimation over lossy networks," Proc. IEEE, vol. 95, pp. 163-187, January 2007.

[6] L. A. Montestruque and P. J. Antsaklis, "Stability of model-based networked control systems with time-varying transmission times," IEEE Trans. Automatic Control, vol. 49, pp. 1562-1572, September 2004.

[7] P. V. Zhivoglyadov and R. H. Middleton, "Networked control design for linear systems," Automatica, vol. 39, pp. 743-750, April 2003.

[8] Y. Ji, H. J. Chizeck, X. Feng and K. A. Loparo, "Stability and control of discrete-time jump linear systems," Control Theory and Advanced Technology, vol. 7, pp. 247-270, 1991.

[9] O. L. V. Costa and M. D. Fragoso, "Stability results for discrete-time linear systems with Markovian jumping parameters," J. Mathematical Analysis and Applications, vol. 179, pp. 154-178, October 1993.

[10] O. C. Imer, S. Yüksel and T. Başar, "Optimal control of LTI systems over unreliable communication links," Automatica, vol. 42, pp. 14291439, September 2006.

[11] S. Hu and W.-Y. Yan, "Stability of networked control systems under a multiple-packet transmission policy," IEEE Trans. Automatic Control, vol. 53, pp. 1706-1711, August 2008.

[12] J. Wu and T. Chen, "Design of networked control systems with packet dropouts," IEEE Trans. Automatic Control, vol. 52, pp. 1314-1319, July 2007.

[13] P. Seiler and R. Sengupta, "An $H_{\infty}$ approach to networked control," IEEE Trans. Automatic Control, vol. 50, pp. 356-364, March 2005.

[14] M. Yu, L. Wang, T. Chu and G. Xie, "Stabilization of networked control systems with data packet dropout and network delays via switching system approach," in Proc. 43rd IEEE Conf. Decision and Control, vol. 4, pp. 3539-3544, Dec. 14-17, 2004.

[15] W.-A. Zhang and L. Yu, "Output feedback stabilization of networked control systems with packet dropouts," IEEE Trans. Automatic Control, vol. 52, pp. 1705-1710, September 2007.

[16] H. Ishii, " $H^{\infty}$ control with limited communication and message losses," Systems and Control Letters, vol. 57, pp. 322-331, April 2008.

[17] A. Sala, "Computer control under time-varying sampling period: An LMI gridding approach," Automatica, vol. 41, pp. 2077-2082, December 2005.

[18] S. Boyd, L. El Ghaoui, E. Feron and V. Balakrishnan, Linear Matrix Inequalities in Systems and Control Theory. Philadelphia, PA: SIAM, 1994.

[19] S. Karlin and H. M. Taylor, A First Course in Stochastic Processes. New York: Academic Press, 2nd ed., 1975. 